\title{
Aplicación de índices vegetales en café (Coffea arabica L.)
}

\section{Application of vegetable indices in coffee (Coffea arabica L.)}

\author{
Edier Fernando Avila-Vélez'; Bibiana Royero-Benavides² \\ IIng. Catastral y Geodesta, Esp., M.Sc. Universidad de Cundinamarca. Fusagasugá - Cundinamarca, Colombia; e-mail: \\ efernandoavila@ucundinamarca.edu.co; (D)https://orcid.org/0000-0002-8997-9125 \\ 2Ing. Agrónoma, M.Sc. Universidad de Cundinamarca. Fusagasugá - Cundinamarca, Colombia; e-mail: broyero@ucundinamarca.edu.co; \\ (D) https://orcid.org/0000-0003-3884-0745
}

Cómo citar: Avila-Vélez, E.F.; Royero-Benavides, B. 2021. Aplicación de índices vegetales en café (Coffea arabica L.). Rev. U.D.C.A Act. \& Div. Cient. 24(2):e1584. http://doi.org/10.31910/rudca.v24.n2.2021.1584

Artículo de acceso abierto publicado por Revista U.D.C.A Actualidad \& Divulgación Científica, bajo una Licencia Creative Commons CC BY-NC 4.0

Publicación o icial de la Universidad de Ciencias Aplicadas y Ambientales U.D.C.A, Institución de Educación Superior Acreditada de Alta Calidad por el Ministerio de Educación Nacional.

Recibido: mayo 31 de 2020

Aceptado: septiembre 23 de 2021

Editado por: Helber Adrián Arévalo Maldonado

\section{INTRODUCCIÓN}

En Colombia, el cultivo de café es uno de los más representativos e importantes económicamente; se estima, que de este cultivo deriva el sustento de más de 540.000 familias, de las cuales, el $96 \%$ son pequeños productores (Cenicafe, 2020).

La caficultura enfrenta importantes retos, debido al fenómeno de cambio climático, que trae consigo sequias prolongadas, temperaturas elevadas o fuertes lluvias, que afectan el desarrollo del cultivo y favorecen problemas fitosanitarios (Jiménez-Torres \& Massa-Sánchez, 2015); por ejemplo, en 2017, Colombia atravezó por una ola invernal, en donde las lluvias, en algunas zonas cafeteras, aumentaron hasta un $35 \%$ más de lo normal, se redujo la temperatura en casi 2 grados y los cafetales recibieron, en un año, 300 horas menos de brillo solar (Cenicafe, 2020).

La agricultura de precisión, se propone como una herramienta para enfrentar las variaciones generadas por el cambio climático y permite la identificación, la cuantificación y el mapeo de la variabilidad espacial y temporal de cultivos (Bongiovanni et al. 2006). El reconocimiento de esta variabilidad, se realiza por medio de la captura de información proveniente de sensores remotos, que discriminan anomalías en el espectro electromagnético y generan alarmas tempranas, que contribuyen en la toma de decisiones de la producción agrícola (Urbano-Molano, 2013).

El monitoreo de la actividad fotosintética, se puede realizar a partir de fotografías digitales multiespectrales, con vehículos aéreos no tripulados, dada su facilidad de operación y planeación de vuelo, permitiendo observar radiométricamente, a través de sensores multiespectrales a bordo, el comportamiento simétrico de la biomasa en el espectro electromagnético (Berrío M. et al. 2015).

Los índices de vegetación, se calculan a partir de los valores de la reflectividad a distintas longitudes de onda, para obtener información relacionada con la vegetación (Gilabert et al. 1997). La combinación de longitudes de onda del espectro electromagnético permite al investigador interpretar el estado saludable o algún tipo de deficiencias en el que se encuentra la vegetación (Ávila Vélez et al. 2019). La condición de la energía clasificada en el espectro electromagnético permite operar algebraicamente la intensidad de reflectividad, con el fin de construir índices espectrales, como el índice normalizado diferencial de vegetación (NDVI, por sus siglas en idioma inglés) (Anyamba \& Tucker, 2005).

La anterior afirmación, se puede describir matemáticamente, analizando las longitudes de onda, de acuerdo con la ecuación 1. 


$$
\lim _{\text {Rojo } \rightarrow 0}\left(\frac{\text { NIR }}{\text { ROJO }}\right) \rightarrow \infty \rightarrow \text { Planta Saludable }
$$

ecuación 1

La ecuación 1 relaciona el estado fotosintético de la biomasa, cuando la fracción tiende a valores máximos, describe una vegetación saludable y cuando la fracción tiende a valores bajos, se evidencia alteraciones de las plantas, debido a algún tipo de estrés, ya sea hídrico, nutricional o sanitario.

Por lo tanto, el propósito del presente estudio fue implementar el monitoreo de la actividad fotosintética en el cultivo de café, mediante índices de vegetación, lo cual, brinda información valiosa relacionada con el crecimiento y la vigorosidad de las plantas, para detectar, de forma temprana, alteraciones que perjudiquen el normal desarrollo del cultivo y facilitar la rápida toma de decisiones (Cruz Flores et al. 2020).

\section{MATERIALES Y MÉTODOS}

Área de estudio. La zona de estudio está localizada en la finca La Portada del municipio de Pandi, Cundinamarca, con una altura aproximada de $1.100 \mathrm{~m}$ s.n.m., una temperatura promedio de $20^{\circ} \mathrm{C}$ y humedad relativa promedio del $67 \%$, con coordenadas 74²7'51" Oeste y 4\%8’3" Norte. El área cercana de sobrevuelo fue de $4.000 \mathrm{~m}^{2}$, en tres lotes de café variedad castillo, con diferentes periodos vegetativos, como se observa en la figura 1.

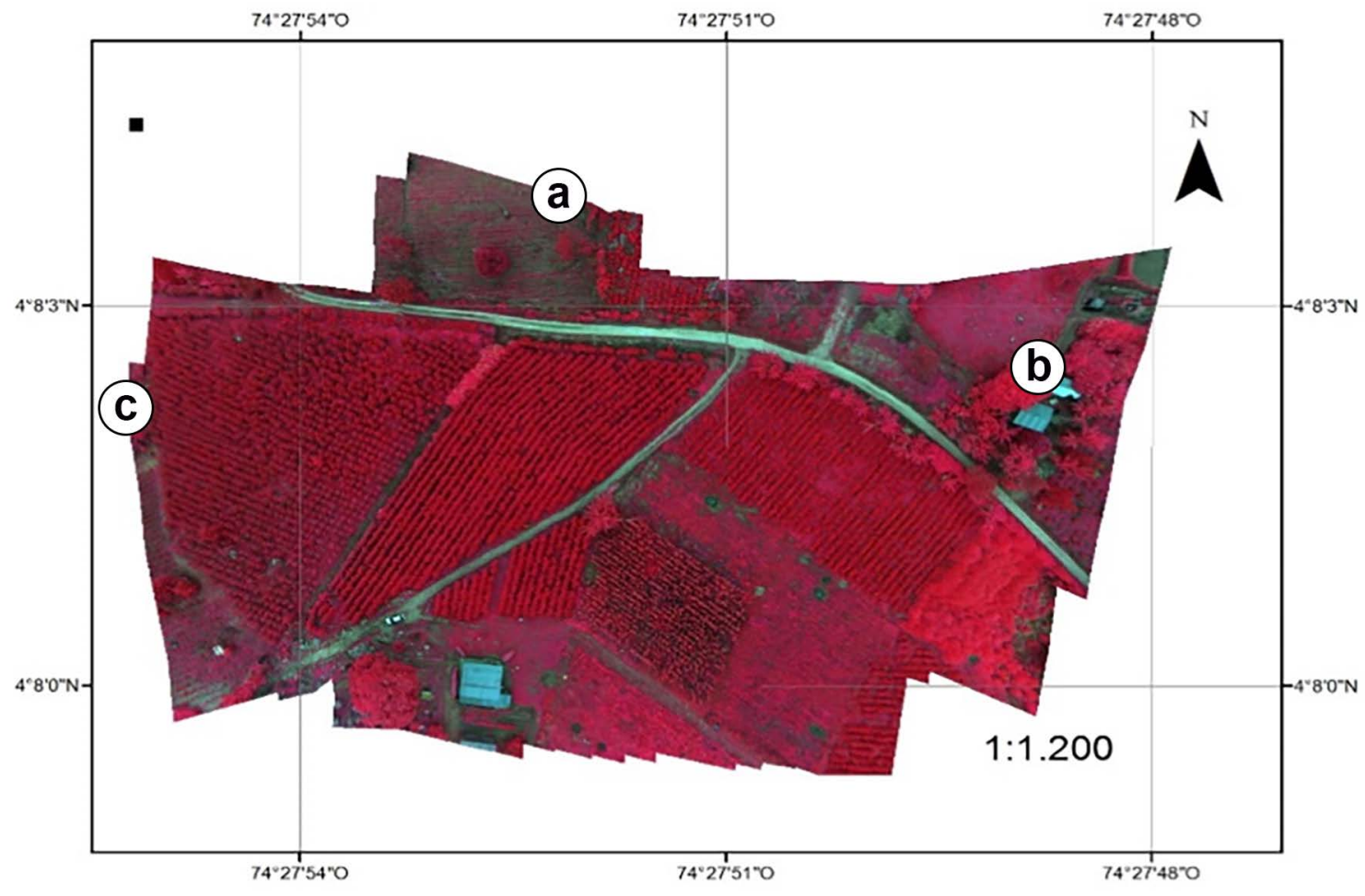

Figura 1. Imagen tomada con el sensor Parrot Sequoia RGB (4-2-1). Lotes con periodo vegetativo de: a. 4 años; b. 2 años; c. 5 años.

Mediante el vehículo aéreo no tripulado Parrot Bluegrass y su sensor multiespectral, se realizó un plan de vuelo en la zona de estudio, utilizando la aplicación Pix4Dcapture, en el que se restituyó las escenas o fotografías tomadas en las diferentes longitudes de onda en el software Pix4Dmapper, para las diferentes bandas, correspondientes a las longitudes de onda del sensor Parrot.

Aplicación de los diferentes índices de vegetación. En el desarrollo de la investigación, se implementaron los siguientes índices de vegetación, con el fin de comparar, radiométricamente, cuáles ofrecen mayor riqueza espectral asociada a la actividad fotosintética del cultivo de café.

$$
\mathrm{NDVI}=\frac{\mathrm{NIR}-\mathrm{ROJO}}{\mathrm{ROJO}+\mathrm{NIR}}
$$

La fórmula del NDVI corresponde al cociente algebraico entre la resta y la suma de las bandas infrarrojo cercano y rojo del sensor Parrot Sequoia.

Este índice fue aplicado por Anyamba \& Tucker (2005), para analizar los cambios del paisaje, utilizando las bandas de infrarrojo cercano y rojo del sensor Spot.

$$
\text { GNDVI }=\frac{\text { NIR }- \text { VERDE }}{\text { NIR }+ \text { VERDE }}
$$

ecuación 3

La ecuación 3, índice normalizado diferencial de vegetación en verde (GNDVI, por sus siglas en idioma inglés), relaciona las bandas del infrarrojo cercano y verde. El presente índice fue aplicado por Gitelson \& Merzlyak (1997), para observar el contenido de la clorofila.

$\mathrm{SAVI}=\frac{\mathrm{NIR}-\mathrm{ROJO}}{(\mathrm{NIR}+\mathrm{ROJO}+\mathrm{L})} \mathrm{X}(1+\mathrm{L})$

ecuación 4 
La ecuación 4, índice de vegetación ajustado al suelo (SAVI, por sus siglas en idioma inglés), oscila entre -1 a 1 y sus valores son adimensionales. Los valores máximos cercanos a uno son el resultado de plantas o cultivos con gran vigorosidad y buen estado vegetativo (Huete, 1988) y los valores cercanos a cero o negativos dan cuenta de malezas, pastos bajos o elementos, sin contenido clorofílico. Se introduce un parámetro L, asociado con la vegetación del suelo en el cultivo.

En la presente investigación, se utilizó una adaptación del índice vegetal de Fitzgerald et al. (2010), denominado índice de clorofila terrestre adaptado (AMTCI, por sus siglas en idioma inglés)

$$
\text { AMTCI }=\left(\frac{\text { BORDE ROJO }- \text { ROJO }}{\text { ROJO }+ \text { VERDE }}\right) \quad \text { ecuación } 5
$$
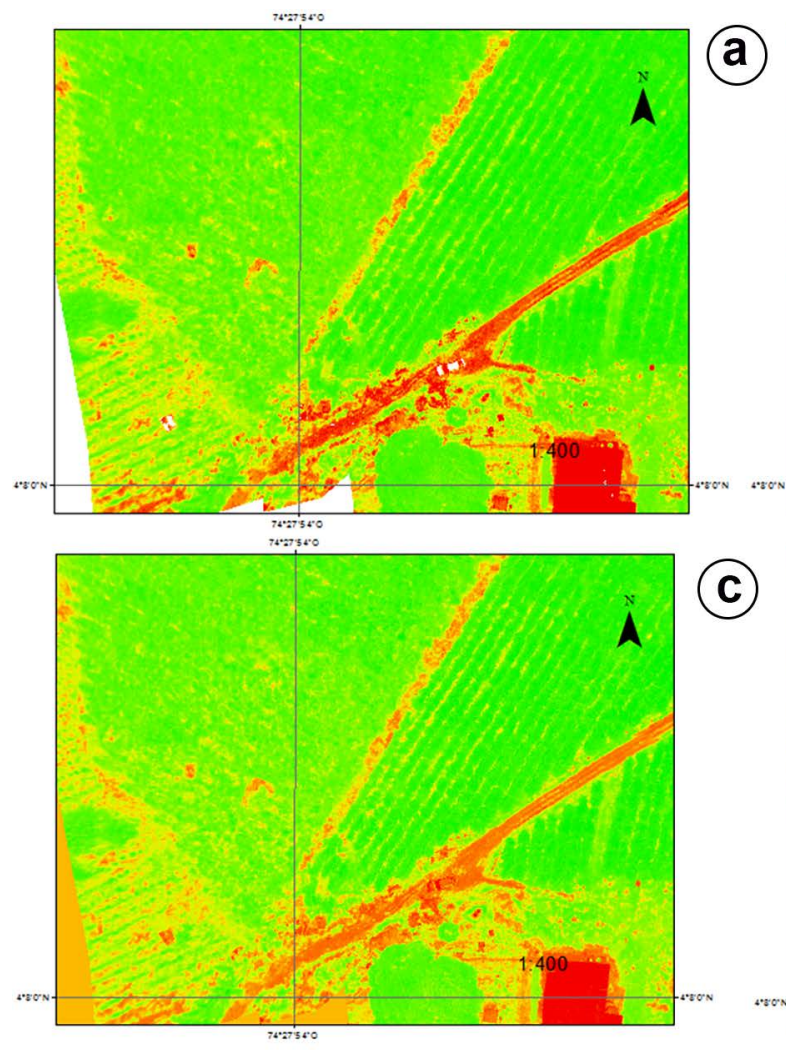

Caracterización de la firma espectral. Se tomaron 5.915 pixeles con resolución espacial de $4 \mathrm{~cm} / \mathrm{px}$, para generar la firma del café arábigo promedio y su respectiva desviación estándar, en las zonas donde se presentaba mayor actividad fotosintética.

\section{RESULTADOS Y DISCUSIÓN}

Con la aplicación de los índices de vegetación NDVI, GNDVI, SAVI y AMTCI, implementados para cuantificar y caracterizar radiométricamente la salud de los cultivos de café, se logró visualizar alteraciones espectrales en algunas zonas del cultivo, especialmente, con la aplicación del índice de vegetación AMCTI (Figura 2).

Con el desarrollo del método, se observa que, para el cultivo de café variedad castillo, dada su composición química y diferente

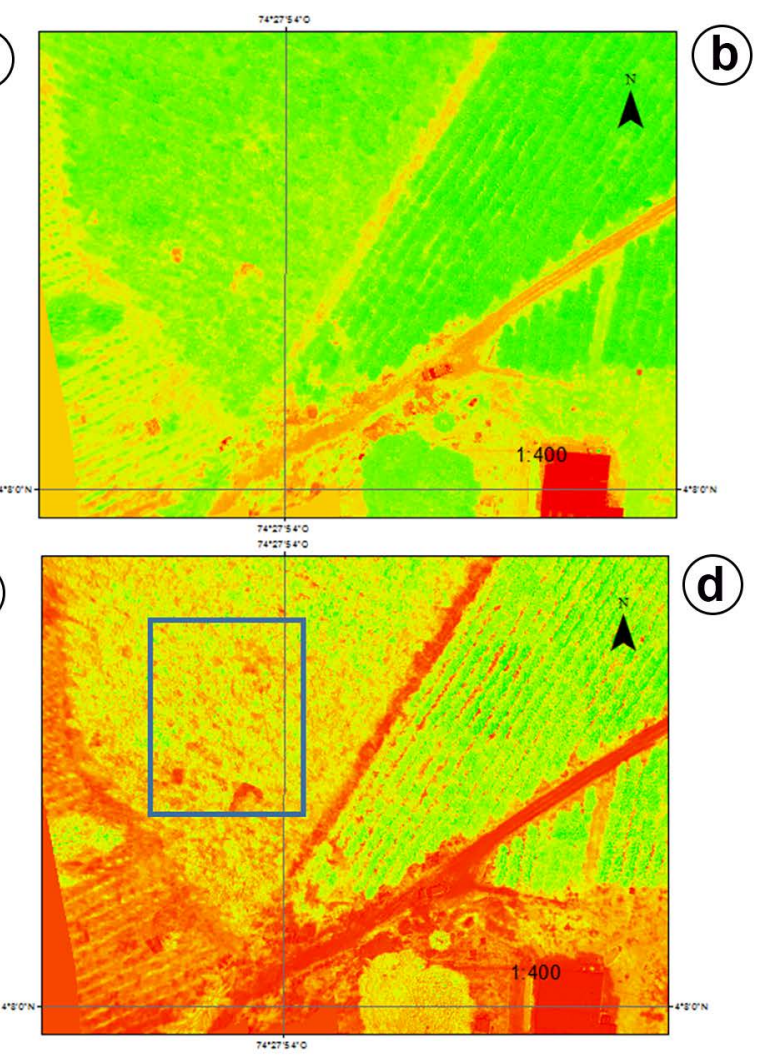

Figura 2. Caracterización espectral detallada de los diferentes índices de vegetación: a. índice normalizado diferencial de vegetación NDVI; b. índice normalizado diferencial de vegetación en verde GNDVI; c. índice de vegetación ajustado al suelo SAVI; d. índice de clorofila terrestre adaptado AMTCI.

temporalidad, el AMCTI muestra una gran variabilidad espectral, como se evidencia en la figura 2; se observa, que los resultados de este índice muestran mayor actividad fotosintética y alta reflectancia, con la banda del Borde Rojo y baja reflectancia, en la banda del Rojo, como se evidencia en el estudio de Gitelson \& Merzlyak (1997).

En la figura 2, se observa en el recuadro azul, que el AMCTI evidencia zonas del cultivo no saludables. Se calcularon las medidas de tendencia central y dispersión, las cuales, indican que el AMCTI posee la mayor variabilidad o desviación estándar espectral de los datos, con un valor de 0,0462; con este parámetro, se evidencian fuertes contrastes fotosintéticos en la vegetación.

El AMCTI propuesto relaciona tres bandas espectrales, lo que conlleva obtener más información fotosintética en las hojas del cultivo de café arábigo. Caso contrario pasa con el NDVI, que posee un punto de saturación, lo que implica acotar la información radiométrica (Schmidt \& Karnieli, 2001). 
Los resultados coinciden con las diferencias evidenciadas en el sistema productivo respecto a su manejo. En el lote c, hay una importante proliferación de arvenses, lo que interfiere en las labores de fertilización y de manejo sanitario. Estas condiciones reducen la tasa del proceso fisiológico de fotosíntesis por debajo de la tasa máxima, respecto de la que podría alcanzar, generando, lo que se puede denominar, como estrés vegetal (Lambers et al. 1998) y se puede relacionar con deficiencias de nitrógeno e irradiancia lumínica (Pérez Asseff et al. 2007), lo que tiende a disminuir el peso seco, el número de hojas y el área foliar (Taiz \& Zeiger, 2002). Otros factores que pueden influir en el establecimiento y en el vigor de la planta, inhibiendo su eficiencia fotosintética, son los de tipo fitosanitario, como plagas, enfermedades y sequía, que son factores de alto impacto en la producción (Pedroza Sandoval et al. 2013).

El valor mínimo para clasificar un cultivo de café con temporalidad de cuatro años y un estado saludable, se puede calcular aplicando el índice de vegetación AMCTI, siendo este valor de 2,44. Esto implica utilizar índices vegetales, para cuantificar los valores mínimos, de posibles afectaciones en proceso productivo (Brizuela-Amador Pérez et al. 2007).

Con el presente estudio, se aportan nuevas técnicas con respecto a la implementación de índices vegetales en el campo de la agricultura de precisión, utilizando el sensor multiespectral Parrot Sequoia, aplicado al cultivo de café. Se debe tener en cuenta, que los cultivos no son homogéneos en sus características fisiológicas y químicas, por consiguiente, se deben realizar estudios, como el presente, para encontrar y caracterizar índices vegetales y firmas espectrales, que determinen el estado fotosintético y alteraciones químicas de las plantas en cada cultivo y temporalidad específica.

El AMCTI presenta la mayor variabilidad espectral entre los índices analizados, siendo el de mayor potencial en el monitoreo y la caracterización de las condiciones fotosintéticas, en el cultivo de café.

Agradecimientos. Los autores agradecen a la Universidad de Cundinamarca, por el apoyo en equipos y al grupo de investigación Geocartografía. Conflicto de intereses: El manuscrito fue preparado y revisado con la participación de todos los autores, quienes declaramos que no existe ningún conflicto de intereses que ponga en riesgo la validez de los resultados presentados.

\section{REFERENCIAS}

1. ANYAMBA, A.; TUCKER, C.J. 2005. Analysis of Sahelian vegetation dynamics using NOAA-AVHRR NDVI data from 1981-2003. Journal of Arid Environments (Estados Unidos). 63(3):596-614. https://doi.org/10.1016/j.jaridenv.2005.03.007

2. ÁVILA VÉLEZ, E.F.; ESCOBAR ESCOBAR, N.; MORANTES CHOCONTA, C.F. 2019. Applying satellite images to spectral signature development of maize production (Zea mays L.) under Colombia's middle tropics conditions.
Entramado (Colombia). 15(2):256-262.

http://dx.doi.org/10.18041/1900-3803/entramado.2.5734

3. BERRÍO M., V.A.; MOSQUERA T., J.; ALZATE V., D.F. 2015. Uso de drones para el análisis de imágenes multiespectrales en agricultura de precisión. Limentech, Ciencia y Tecnología Alimentaria (Colombia). 13(1):28-40.

https://doi.org/10.24054/16927125.v1.n1.2015.1647

4. BONGIOVANNI, R.; MANTOVANI, E.C.; BEST, S.; ROEL, A. 2006. Agricultura de precisión: integrando conocimientos para una agricultura moderna y sustentable. Procisur/IICA. 246p.

5. BRIZUELA-AMADOR PÉREZ, B.; ALCÁNTARGONZÁLEZ, G.; SÁNCHEZ-GARCÍA, P.; PEA-KALRA, Y.; CRUMBAUGH, J.; OLIVE, C.; TIJERINA-CHÁVEZ, L.; MALDONADO-TORRES, R. 2007. Establecimiento de índices espectrales en el diagnóstico nutrimental de nitrógeno en maíz. Agrociencia (México). 41(8):827-835.

6. CENTRO NACIONAL DE INVESTIGACIONES DE CAFÉ, CENICAFE. 2020. Informe anual Cenicafé 2020. https://doi.org/10.38141/10783/2020

7. CRUZ FLORES, D.D.; CURBELO BENÍTEZ, E.A.; FERRER SÁNCHEZ, Y.; ÁVILA, D.D. 2020. Variaciones espaciales y temporales en el Índice de Vegetación de Diferencia Normalizada en Cuba. Ecosistemas. Ecosistemas Revista Científica de Ecología y Medio Ambiente. 29(1):1885. https://doi.org/10.7818/ECOS.1885

8. FITZGERALD, G.; RODRIGUEZ, D.; O'LEARY, G. 2010. Measuring and predicting canopy nitrogen nutrition in wheat using a spectral index-The canopy chlorophyll content index (CCCI). Field Crops Research (Holanda). 116(3):318-324. https://doi.org/10.1016/j.fcr.2010.01.010

9. GILABERT, M.A.; GONZÁLEZ-PIQUERAS, J.; GARCÍAHARO, J. 1997. Acerca de los índices de vegetación. Revista de teledetección. 8:1-10.

10. GITELSON, A.A.; MERZLYAK, M.N. 1997. Remote estimation of chlorophyll content in higher plant leaves. International Journal of Remote Sensing (Reino Unido). 18(12):2691-2697.

https://doi.org/10.1080/014311697217558

11. HUETE, A.R. 1988. A soil-adjusted vegetation index (SAVI). Remote Sensing of Environment. 25(3):295-309. https://doi.org/10.1016/0034-4257(88)90106-X

12. JIMÉNEZ-TORRES, A.; MASSA-SÁNCHEZ, P. 2015. Producción de café y variables climáticas: El caso de Espíndola, Ecuador. Economía. 40:117-137. 
13. LAMBERS, H.; CHAPIN, F.S.; PONS, T.L. 1998. Plant physiological ecology. Springer-Verlag Inc, New York. 540p.

14. PEDROZA SANDOVAL, A.; TREJO CALZADA, R.; CHÁVEZ RIVERO, J.A.; SAMANIEGO GAXIOLA, J.A. 2013. Tolerancia al estrés hídrico y fitosanitario mediante indicadores agronómicos y fisiológicos en diferentes variedades de frijol (Phaseolus vulgaris L.). Revista Mexicana de Fitopatología (Mexico). 31(2):91-104.

15. PÉREZ ASSEFF, J.M.; PEÑA SALAMANCA, E.J.; TORRES GONZÁLEZ, C. 2007. Efecto del nitrógeno y la irradianza en la eficiencia fotosintética del Anamú Petiveria alliacea (Phytolaccaceae). Rev. Academia Colombiana de Ciencias Exactas, Físicas y Naturales. Nacional (Colombia). 31(118):49-55.
16. SCHMIDT, H.; KARNIELI, A. 2001. Sensitivity of vegetation indices to substrate brightness in hyper-arid environment: the Makhtesh Ramon Crater (Israel) case study. International Journal of Remote Sensing. 22(17):3503-3520. https://doi.org/10.1080/01431160110063779

17. TAIZ, L.; ZEIGER, E. 2002. Photosynthesis: physiological and ecological considerations. In: Taiz, L.; Zeiger, E. (eds.). Plant Physiology. Fourth edition. p.172-174.

18. URBANO-MOLANO, F.A. 2013. Redes de sensores inalámbricos aplicadas a optimización en agricultura de precisión para cultivos de café en Colombia. Journal de Ciencia e Ingeniería. 5(1):46-52. 